\title{
¿QUÉ PENSAMOS DE LA INVESTIGACIÓN EN TERAPIA OCUPACIONAL? PERCEPCIÓN DE UN GRUPO DE ESTUDIANTES Y DOCENTES EN CHILE
}

\author{
WHAT WE THINK ABOUT OCCUPATIONAL THERAPY RESEARCH? PERCEPTIONS \\ FROM A GROUP OF ACADEMICS AND STUDENTS IN CHILE
}

\section{José Aravena C. ${ }^{1}$, Jean Gajardo J. ${ }^{2}$ y Alexa Molina $Q .{ }^{3}$}

\begin{abstract}
Resumen
La investigación científica es la base del conocimiento para las disciplinas de la salud. En Terapia ocupacional ha tenido un desarrollo creciente y prioritario, por lo que se hace necesario comenzar a conocer las percepciones de los propios terapeutas en relación a este tema. Para conocer cuál es la percepción que tienen los docentes y estudiantes en relación a la investigación en terapia ocupacional se realizó un estudio descriptivo transversal en un grupo de estudiantes (11) y docentes (17) de una Escuela de Terapia ocupacional entre septiembre 2015 y enero 2016. Se les aplicó un cuestionario presencial que contenía: antecedentes sociodemográficos, grado académico, realización de cursos relacionados a investigación, grado de interés hacia al investigación en Terapia ocupacional e importancia de esta, y conocimiento de conceptos relacionados a lo basado en la evidencia. Se analizaron los datos de forma descriptiva y las preguntas abiertas mediante respuestas acumuladas según categorías. El 86,5\% de las personas manifestó un alto interés en la investigación, un 50\% conocía el concepto de Terapia ocupacional basada en evidencia (TOBE), y los temas más relevantes que generan interés e importancia de la investigación son el poder validar y posicionar el rol del terapeuta ocupacional, y generar conocimiento que permita fortalecer la práctica. En conclusión, tanto los estudiantes como los docentes refieren interés por aprender de investigación en terapia ocupacional y su aplicación. Se hace necesario incorporar conceptos como el de TOBE, y reforzar la educación de pre y posgrado en investigación dirigida a la profesión.
\end{abstract}

\section{Palabras claves:}

Terapia Ocupacional; Practica Basada en la Evidencia; Investigación.

\begin{abstract}
Scientific research is the basis of knowledge for health disciplines. In occupational therapy has had a growing and priority development, so it is necessary to begin to understand the perceptions of therapists themselves in relation to that topic. To know what is the perception that teachers and students in relation to occupational therapy research descriptive cross-sectional study was conducted in a group of teachers and students from a School of Occupational Therapy between September 2015 and January 2016. They were applied a face questionnaire containing: sociodemographic background, academic degree, conducting research related courses, degree of interest in the research in occupational therapy and importance of this, and understanding of concepts related to evidence-based. The data were analyzed descriptively and the open questions by accumulated responses according to categories. $86.5 \%$ of people expressed a strong interest in research, 50\% knew the concept of evidence-based Occupational Therapy (TOBE), and the most relevant topics that generate interest and importance of the research are to validate and positioning the role of the occupational therapist, and generate knowledge to strengthen practice. In conclusion, both students and teachers refer interest in learning from occupational therapy research and application. It is necessary to incorporate concepts such as $T O B E$, and strengthen education in undergraduate and graduate research aimed at the profession.
\end{abstract}

\section{Keyword:}

Occupational Therapy; Evidence-Based Practice; Research.

Fecha de recepción: 09/09/2016

Fecha aceptación: 21/11/2016

1 Licenciado en Ciencia de la Ocupación, Universidad de Chile. Magister en Envejecimiento y Calidad de vida, INTA, Universidad de Chile. Escuela de Terapia Ocupacional, Universidad Bernardo O’higgins. Correo: jmiguel.aravena@ubo.cl.

2 Licenciado en Ciencia de la Ocupación, Universidad de Chile. Master en Gerontología. Candidato a Doctor en Salud Pública, Universidad de Chile. Departamento de Terapia ocupacional, Universidad de Chile. Correo: jean.gajardo@uchile.cl.

Licenciada en Terapia ocupacional, Universidad Central. Correo: alexa.molina22@gmail.com. 


\section{INTRODUCCIÓN}

A pesar de ser una disciplina joven, la Terapia ocupacional se ha desarrollado a lo largo de los años evolucionando en sus praxis y en los enfoques utilizados para sustentar sus bases, generando un marco de conocimiento en base a la investigación que permita delinear y entregarle una visión al actuar de la Terapia ocupacional (Kielhofner, 2006).

Sin embargo, aún se hace necesario un cuerpo contundente de literatura científica en relación a las bases teóricas e intervención de Terapia ocupacional, para interrumpir la incertidumbre existente en relación a los verdaderos resultados y beneficios que puede traer una intervención desde la mirada de la ocupación, lo que ha despertado la necesidad de posicionar la investigación en Terapia ocupacional como un elemento fundamental para resolver dichas interrogantes (Gustafsson, Molineux, \& Bennett, 2014).

Ante esto, en el año 2011 la American Journal of Occupational Therapy estableció la agenda de prioridades en relación a la investigación en la profesión, describiendo cinco puntos clave: 1) medición/evaluación, 2) investigación en intervenciones, 3) investigación básica, 4) investigación traslacional, 5) socialización de la profesión, y 6) formación de profesores y recursos académicos (American Occupational Therapy Association, 2011). De estos puntos se desprendió la importancia de la educación en Terapia ocupacional y su vinculación con la investigación, como un medio para alcanzar dichas prioridades. Es por esto que en el año 2014, se estableció la agenda de prioridades en relación a la investigación en educación en Terapia ocupacional, mencionando como tópicos relevantes la construcción de teorías, la pedagogía, métodos educativos, características y competencias del aprendizaje, socialización de la profesión, y formación de profesores y recursos académicos (American Occupational Therapy Association, 2014).

Ya estableciendo la prioridad que tiene la investigación disciplinar en Terapia ocupacional diversos estudios han tratado de conocer sobre la percepción de estudiantes y profesionales en relación a la investigación y las practicas basadas en la evidencia en Terapia ocupacional. Una revisión sistemática conducida en el 2014 resumió como principales hallazgos en 32 estudios incluidos, que en general los profesionales Terapeutas ocupacionales presentan una buena percepción y motivación hacia la investigación y la práctica basada en la evidencia, pero que encuentran diversas barreras para su uso e implementación, mencionando el conocimiento en relación a la investigación o como implementar la práctica basada en la evidencia en su práctica cotidiana como principales necesidades (Upton, Stephens, Williams, \& Scurlock-Evans, 2014).

Conocer en relación a las percepciones y necesidades relacionadas a la investigación y prácticas basas en la evidencia ha permitido desarrollar diversos programas de entrenamiento y capacitación para estudiantes y profesionales de la salud (Thomas, Saroyan, \& Dauphinee, 2011). Esto no ha sido ajeno para la Terapia ocupacional, donde también se han testeado diversos programas para la facilitación de la incorporación de conocimientos relacionados a investigación en estudiantes y profesionales, producto de la necesidad creciente de incorporar un conocimiento científico en la práctica y entendimiento de la disciplina (Cohn, Coster, \& Kramer, 2014; du Toit, Wilkinson, \& Adam, 2010; Vogel, 2012).

En Chile y en Latinoamérica este ha sido un tema escasamente explorado, emergiendo la necesidad de conocer cuál es la percepción que tienen los profesionales y estudiantes en relación a la investigación en Terapia ocupacional, con el fin de rescatar las necesidades locales en relación a este tema. Es por esto que el objetivo de este estudio fue conocer y describir cuál es la percepción que tienen los docentes y estudiantes de una escuela de la Terapia ocupacional, en relación a la investigación y las prácticas basadas en la evidencia en Terapia ocupacional.

\section{MATERIAL Y MÉTODOS}

Estudio con diseño transversal de carácter descriptivo, efectuado en la Escuela de Terapia Ocupacional de la Universidad Bernardo O'Higgins entre los meses de septiembre del año 2015 y enero del año 2016. Corresponde a un estudio con metodología cuantitativa, que busca describir cual es la percepción y conocimiento en relación a la investigación y prácticas basadas en la evidencia en Terapia ocupacional, que tienen un grupo de docentes y estudiantes.

Para el estudio se seleccionaron dos muestras por conveniencia. Una muestra correspondió a estudiantes de cuarto año de la carrera de Terapia ocupacional que se encontraban cursando el ramo de "Metodología de 
la Investigación I" en septiembre del 2015. La segunda muestra correspondió a académicos y docentes de campos clínicos de la Escuela de Terapia Ocupacional que participaron de una jornada de capacitación en "Investigación en Terapia ocupacional" en enero del 2016.

\section{Recolección de información}

Los datos fueron recolectados en dos ocasiones: en un primer momento con los estudiantes y otra con los docentes.

Primera instancia (estudiantes): Durante su cuarta semana de clases en el ramo de Metodología de la investigación, antes de comenzar la clase se les invito a contestar voluntariamente una entrevista auto-administrada relacionada a su percepción hacia la investigación y evidencia en Terapia ocupacional. Dicha administración tuvo un tiempo aproximado de 15 minutos.

Segunda instancia (docentes): durante una jornada de capacitación a docentes y académicos de la Escuela de Terapia ocupacional, antes de comenzar la capacitación se invitó a los docentes a contestar voluntariamente una entrevista auto-administrada relacionada a su percepción hacia la investigación y evidencia en Terapia ocupacional. Dicha administración también tuvo una duración aproximada de 15 minutos.

\section{Variables de estudio}

Para este estudio se recolectaron las siguientes variables

Variables de los estudiantes: antecedentes sociodemográficos (edad, sexo), grado académico más alto, años estudiando Terapia ocupacional, cursos de pregrado relacionados a investigación cursados.

Variables de los docentes: antecedentes sociodemográficos (edad, sexo), grado académico más alto, cursos de postítulo en investigación, participación en investigación en el último año. A través de preguntas dicotómicas se les consulto si se sentían preparados para realizar investigación en terapia ocupacional y si les gustaría aprender más en relación a cómo realizar investigación disciplinar.

Variables para ambos sujetos: interés hacia la investigación a través de pregunta con respuesta ordinal (muy bajo interés - muy alto interés), conocimiento de los conceptos de Medicina basada en la evidencia $(\mathrm{MBE})$, Práctica basada en la evidencia (PBE), y Terapia ocupacional basada en la evidencia (TOBE), a través de preguntas con respuesta dicotómica. Además, a través de dos preguntas abiertas se recolectó información en relación a: porque tiene interés en aprender de investigación en terapia ocupacional (en relación a los que manifestaron interés en la investigación), y porque cree que es importante la investigación en Terapia ocupacional.

\section{Análisis de datos}

Para el análisis de datos, en primera instancia se realizó una descripción de los sujetos según datos sociodemográficos y antecedentes relacionados a su participación en instancias vinculadas a la investigación en su formaicíón, considerando medidas de tenencia central y dispersión (promedio, desviación estándar, mínimo, máximo) para las variables continuas y porcentajes, gráficos y tablas de frecuencia par las variables ordinales y dicotómicas (interes hacia la investigación y conocimiento de conceptos).

Las preguntas abiertas ("ipor qué considera improtante la investigación en Terapia ocupacional?" y "¿por qué le interesa realizar investigacion en Terapia ocupacional?"), fueron analizadas como encuesta con pregunta abierta, categorizando las respuestas más frecuentes para luego comparar su frecuencia y porcentaje a traves de una tabla de respuestas acumuladas.

\section{Consideraciones éticas}

Para las consideraciones éticas de este trabajo, se le ha otorgado a cada participante un consentimiento informado, en donde se da a conocer que toda la información recopilada será de uso exclusivo de los autores de este estudio, el cual fue conferido antes de realizar la encuesta. Tanto los docentes como los estudiantes accedieron a participar de manera voluntaria. El uso de sus datos personales fue confidencial y exclusivo para realizar éste trabajo de investigación y la información recolectada en las encuestas es de carácter anónimo.

\section{Resultados}

En la Tabla 1 se describe la muestra total de participantes (28 personas), donde el promedio de edad fue de 27 años, con un 79\% (22 personas) de mujeres. De los estudiantes ( 11 personas) el promedio de edad fue de 22 años, 82\% (9 personas) fueron mujeres. En pro- 
medio llevaban 4 años estudiando Terapia ocupacional, y el $55 \%$ (6 personas) ya había realizado cursos de pregrado relacionados con investigación.

De los docentes (17 personas), el promedio de edad fue de 30 años, 76\% (13 personas) correspondieron a mujeres, y donde el $47 \%$ (8 personas) tenía como grado académico más alto el de licenciado y el $41 \%$ (7 personas) grado de magister. El 18\% (3 personas) respondieron contar con cursos de postítulo relacionados con investigación, y el 24\% (4 personas) de los docentes afirmó haber participado en alguna investigación en el último año.

Tabla 1.

Estadísticos descriptivos de la muestra de docentes y estudiantes.

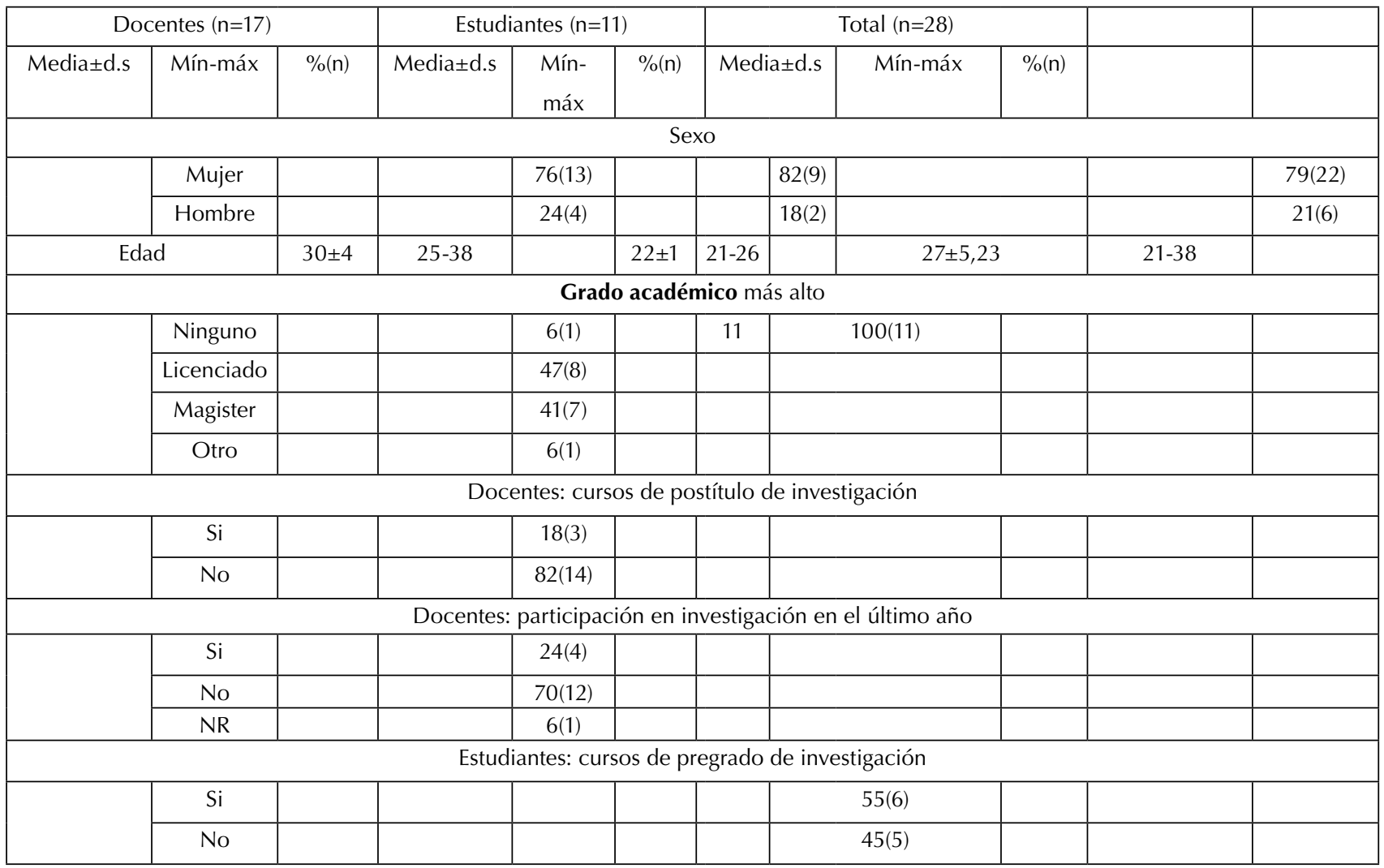

D.S desviación estándar, Mín mínimo

Máx máximo, \% porcentaje

n número de sujetos.

\section{Interés en aprender de investigación en Terapia ocupacional}

En el gráfico 1 se muestra la distribución de docentes y estudiantes según el nivel de interés reportado en relación a aprender de investigación en Terapia ocupacional. El $100 \%$ de los sujetos respondió tener entre regular y muy alto interés hacia la investigación disciplinar $(13,5 \%$ regular interés, $48 \%$ un alto interés, y un $38,5 \%$ un muy alto interés).
De los docentes, el 18\% respondió tener regular interés en aprender de investigación en la disciplina, el $41 \%$ un alto interés, y el $41 \%$ un muy alto interés. Por otra parte, de los estudiantes, el 9\% respondió tener regular interés en aprender de investigación en Terapia ocupacional, $55 \%$ un alto interés, y un $36 \%$ un muy alto interés. 


\section{Gráfico 1:}

Descripción del porcentaje de docentes y estudiantes en relación al nivel de interés hacia la investigación en Terapia ocupacional.

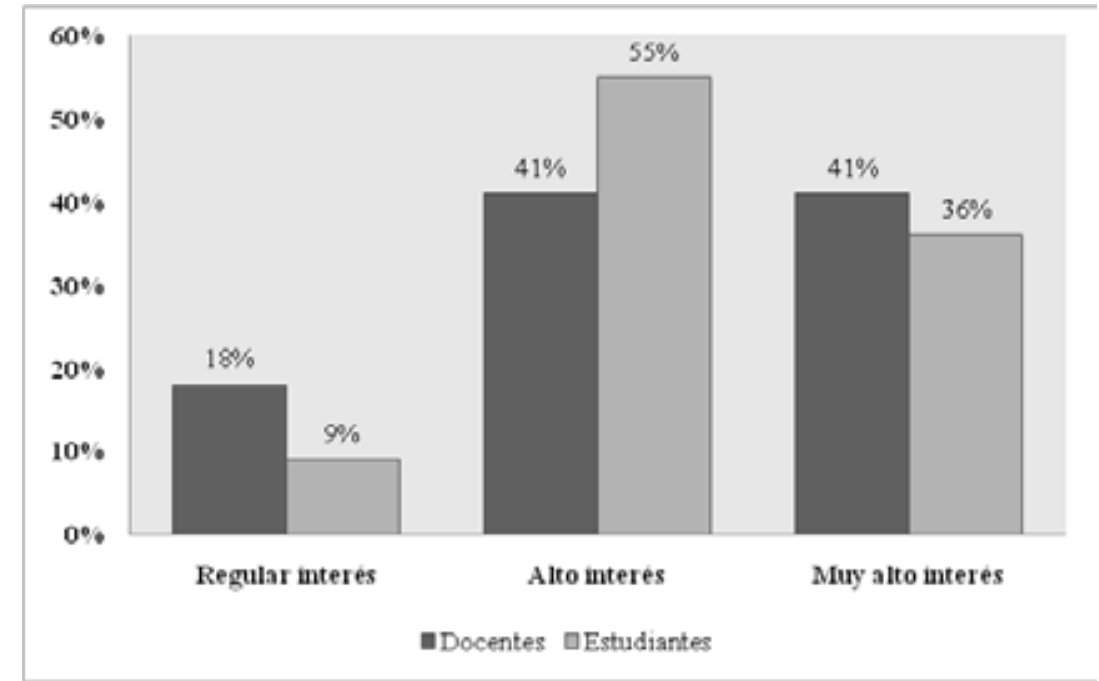

Conocimientos en relación a conceptos de "basado en la evidencia"

El gráfico 2, ilustra la frecuencia encontrada en relación al conocimiento existente de los términos $\mathrm{MBE}$, PBE, y TOBE entre los entrevistados. Del total de participantes, el 54\% refiere conocer el concepto de MBE
(65\% docentes; $36 \%$ estudiantes), un $71 \%$ conoce el concepto de PBE (65\% docentes; $82 \%$ estudiantes), y un $50 \%$ reconoce el termino TOBE (53\% docentes; $55 \%$ estudiantes).

\section{Gráfico 2:}

Descripción del porcentaje de docentes y estudiantes que afirma conocer los conceptos de MBE, PBE y TOBE.

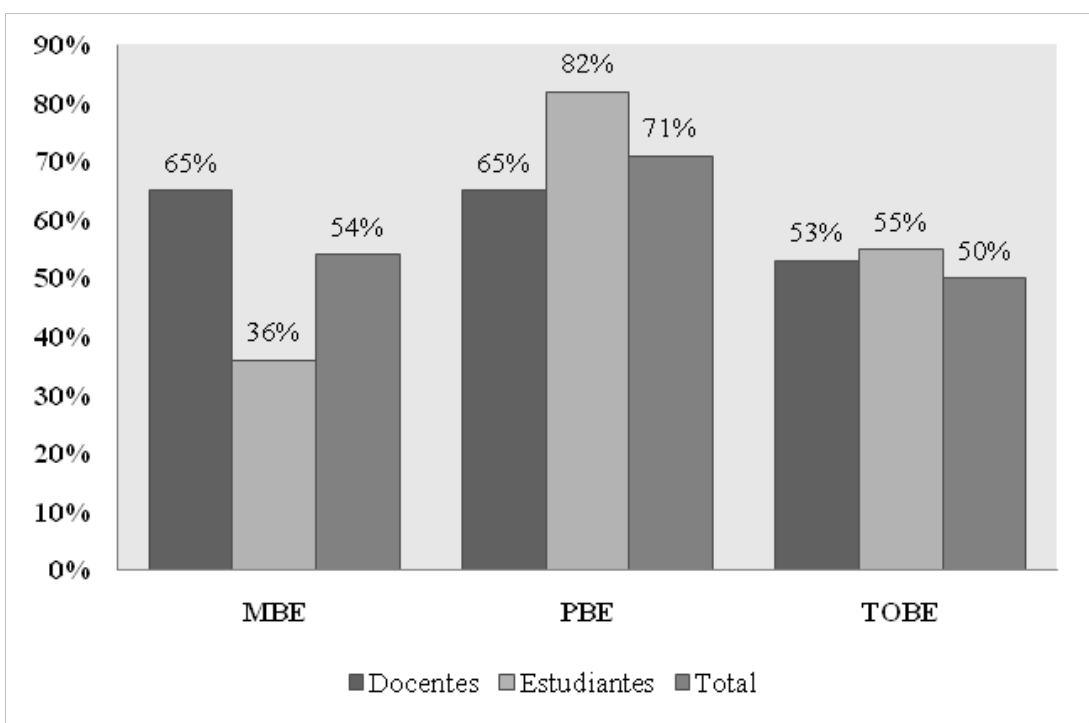

MBE medicina basada en evidencia, PBE práctica basada en evidencia, TOBE Terapia ocupacional basada en evidencia. 
En cuanto a la pregunta realizada tanto a docentes como estudiantes en referencia a si desea aprender acerca del uso de la evidencia en Terapia ocupacional, el 100\% de los sujetos (28 personas) responde de forma afirmativa.

Por otra parte, de los docentes, el 59\% (10 personas) afirman que se sienten capacitados para realizar una investigación. Además el $100 \%$ de los docentes (11 personas) señala que les gustaría aprender más acerca de cómo realizar una investigación en terapia ocupacional.

\section{Importancia e intereses hacia la investigación en Terapia ocupacional}

Para la muestra total, las respuestas más frecuentes relacionadas con el interés que presentaban los sujetos para realizar investigación disciplinar son el poder vali- dar o posicionar el rol del Terapeuta ocupacional (18\% docentes; $45 \%$ estudiantes), el generar conocimiento nuevo y evidencia que aporte a la profesión (23\% docentes; $36 \%$ estudiantes), mejorar el curriculum vitae (CV) o la formación profesional con respecto al tema ( $23 \%$ docentes; $18 \%$ estudiantes), y el aporte y crecimiento de la profesión (18\% de docentes; $45 \%$ estudiantes) (tabla 2).

Las respuestas más frecuentes relacionadas a porque es importante la investigación en Terapia ocupacional, del total de la muestra se puede encontrar el poder validar o posicionar el rol del Terapeuta ocupacional (35\% docentes; $36 \%$ estudiantes), el generar conocimiento nuevo y evidencia que aporte a la profesión (35\% docentes; $45 \%$ estudiantes), y el protocolizar, sistematizar y sustentar el conocimiento de Terapia ocupacional (23\% docentes; $36 \%$ estudiantes) (tabla 2 ).

Tabla 2.

Tópicos más frecuentes de respuestas de docentes y estudiantes relacionados con la importancia e interés acerca de la investigación en T.O.

\begin{tabular}{|c|c|c|c|}
\hline & Respuestas & $\begin{array}{l}\text { Interés hacia la investiga- } \\
\text { ción en TO \%(n) }\end{array}$ & $\begin{array}{l}\text { Importancia de la inves- } \\
\text { tigación en TO \%(n) }\end{array}$ \\
\hline \multirow{9}{*}{ Docentes } & Quehacer profesional de T.O. & $5,8 \%(1)$ & $18 \%(3)$ \\
\hline & Validar/ demostrar/ posicionar/ el rol del T.O. & $18 \%(3)$ & $35 \%(6)$ \\
\hline & Aporte/ crecimiento de la profesión. & $18 \%(3)$ & $23 \%(4)$ \\
\hline & $\begin{array}{c}\text { Generar conocimiento/ desarrollo/ nuevo/ innovación/ evidencia/ aporte } \\
\text { a la T.O. }\end{array}$ & $23 \%(4)$ & $35 \%(6)$ \\
\hline & Ampliar/ abrir campo en diversas áreas de la profesión. & 0 & $12 \%(2)$ \\
\hline & Calidad de la atención brindada por T.O. & 0 & $12 \%(2)$ \\
\hline & Mejorar intervención/ nutrir conocimiento de T.O. & $12 \%(2)$ & $5,8 \%(1)$ \\
\hline & Protocolizar/ Sistematizar/ sustentar el conocimiento de T.O. & 0 & $23 \%(4)$ \\
\hline & Mejorar el CV/ Formación personal respecto a la carrera. & $23 \%(4)$ & 0 \\
\hline \multirow{9}{*}{ Estudiantes } & Quehacer profesional del T.O. & $27 \%(3)$ & $18 \%(2)$ \\
\hline & Validar/ demostrar/ posicionar/ el rol del T.O. & $45 \%(5)$ & $36 \%(4)$ \\
\hline & Aporte/ crecimiento de la profesión. & $45 \%(5)$ & $18 \%(2)$ \\
\hline & $\begin{array}{c}\text { Generar conocimiento/ desarrollo/ nuevo/ innovación/ evidencia/ aporte } \\
\text { a la T.O. }\end{array}$ & $36 \%(4)$ & $45 \%(5)$ \\
\hline & Ampliar/ abrir campo en diversas áreas de la profesión. & 0 & $18 \%(2)$ \\
\hline & Mejorar intervención/ nutrir conocimiento de la carrera. & $18 \%(2)$ & 0 \\
\hline & Protocolizar/ Sistematizar/ sustentar el conocimiento de T.O. & 0 & $36 \%(4)$ \\
\hline & Mejorar el CV/ Formación personal. & $18 \%(2)$ & 0 \\
\hline & Autocrítica/ reflexión respecto a la profesión y desempeño de la misma. & $9 \%(1)$ & $9 \%(1)$ \\
\hline
\end{tabular}

TO, Terapia ocupacional

\section{CV, Curriculum vitae}




\section{Discusión}

En esta investigación se buscó conocer cuál era la percepción que tenían un grupo de docentes y estudiantes de la Escuela de Terapia ocupacional de la Universidad Bernardo O'Higgins en relación a la investigación en la profesión y su interés hacia ella. De los resultados generales se puede observar que tanto estudiantes como docente tenían una percepción bastante positiva hacia la investigación, presentando en su mayoría un alto interés en aprender más aspectos vinculados a su entendimiento e implementación. Estos resultados van en relación con los obtenidos en estudios realizados en otros contextos, lo que reafirma el interés y necesidad que tienen los Terapeutas ocupacionales de incorporar más elementos de la investigación científica en su formación y quehacer profesional (Karlsson \& Törnquist, 2007; Lyons, Brown, Tseng, Casey, \& McDonald, 2011; Thomas \& Law, 2014).

En relación a porque les interesa aprender de investigación en Terapia ocupacional, las respuestas más frecuentes fueron el poder validar o posicionar el rol del Terapeuta ocupacional, el generar conocimiento nuevo y evidencia que aporte a la profesión, y mejorar el curriculum vitae (CV) o la formación profesional con respecto al tema. Para la pregunta porque es importante, las respuestas más frecuentes fueron el poder validar o posicionar el rol del Terapeuta ocupacional, el generar conocimiento nuevo y evidencia que aporte a la profesión, y el protocolizar y sistematizar la intervención. Al parecer el mayor interés e importancia que le atribuyen los Terapeutas ocupacionales en formación y ya egresados a la investigación en la disciplina es el poder validar posicionar el rol que tiene el Terapeuta ocupacional. Este hallazgo podría reflejar el punto en el que se halla el desarrollo de la profesión actualmente en Chile, donde aún se encuentran en discusión temas como "que es la terapia ocupacional", "que es la ciencia de la ocupación", y cuál es el rol del terapeuta ocupacional en los diversos contextos de trabajo. Ante esto llama la atención que se entienda la investigación de forma primaria como una forma de posicionamiento y de validación del quehacer profesional y personal (asumiendo que el quehacer actual es el correcto), por sobre aspectos como el aumentar los conocimientos para mejorar o evaluar las prácticas actuales o para generar solución a dichas interrogantes que aún están en discusión, como se ha encontrado en otros estudios realizados en otros contextos (Karlsson \& Törnquist, 2007). De todas formas el interés de generar nuevo conocimiento y evidencia que aporte a la profesión se mantiene como una razón importante para seguir construyendo conocimiento científico en Terapia ocupacional.

Otro hallazgo que Ilama la atención es que tan capacitados se sienten los docentes para llevar a cabo un proyecto de investigación. El 59\% afirma sentirse capacitado para llevar a cabo un proyecto de investigación. Dicho dato toma relevancia cuando se considera que el $47 \%$ de ellos afirma tener grado académico de magister, por lo que no sólo tener dicho grado académico se relaciona con sentirse competente para ejecutar una investigación, sino que más bien tendría que ver con las capacidades que percibe para ejecutar un proyecto e interpretar sus resultados de manera científica (Fänge \& Ivanoff, 2009). Aunque dicha hipótesis se contrapone al evidenciar que sólo el 18\% (3 personas) respondieron contar con cursos de postítulo relacionados con investigación, lo que hace pensar en relación a la formación de magister por la que optan los Terapeutas ocupacionales, la cual podría contener escaso contenido de formación en investigación, a pesar de que finalizar un magister conlleva un proyecto de tesis (que serpia un proyecto de investigación), priorizando la entrega de conocimiento profesional por sobre el académico. Y en correlato con estos hallazgos, se observa que sólo el $24 \%$ (4 personas) de los docentes afirmó haber participado en alguna investigación en el último año, lo que indica que un bajo porcentaje de los Terapeutas ocupacionales está participando de generación de conocimiento profesional u de otro carácter. De todas formas se espera que esta observación se haya dado de esta forma puesto sólo se indago en un grupo de docentes de una sola Universidad.

En el área donde se observaron más equiparadas las respuestas de ambos grupos fue en los conocimientos de conceptos ligados al uso de la evidencia. Para todas las respuestas, no existían categorías predominantes entre los conceptos conocidos; destaca que el concepto más conocido por los docentes es el de medicina basada en la evidencia $(65 \%)$ y práctica basada en la evidencia (65\%), y para los estudiantes el de práctica basada en la evidencia (82\%). Sin embargo el concepto que fue menos familiar para ambos grupos (los estudiantes lo reportaron como más familiar) fue el de Terapia ocupacional basada en la evidencia (TOBE). Esto puede deberse a que este concepto ha tomado fuerza durante los últimos siete u ocho años, por lo que es más probable que los estudiantes estén más familiarizados puedo puede haber sido incorporado ya dentro de su formación profesional. No así en el caso de los docen- 
tes, donde algunos pueden tener más años de egresados, por lo que este concepto puede no ser tan familiar. De todas formas este es un punto importante puesto si los docentes no conocen dicho concepto, podría inferirse que el uso de la evidencia científica en conjunto con las preferencias del usuario como elementos para la toma de decisiones en los tratamientos de Terapia ocupacional (Thomas \& Law, 2013), no es una práctica habitual del ejercicio profesional local. Aunque para llegar a dicha inferencia es necesario ahondar de forma más precisa y ampliada en el conocimiento y uso de dicha práctica en Chile.

La principal limitación de este estudio es que fue realizado sólo con un grupo de estudiantes, que para ese entonces representaban cerca del nueva a $10 \%$ del total de estudiantes que cursaban por Terapia ocupacional, y de docentes de una Escuela de Terapia ocupacional, por lo que sus resultados se hacen difícilmente extrapolables a todos los contextos académicos de formación en Chile. De todas formas la muestra de docentes es de un carácter más heterogéneo, puesto muchos de ellos podrían haber tenido formaciones distintas y años de ejercicio profesional diversos, por lo que de todas formas, los resultados encontrados en este estudio permiten realizar un primer levantamiento y conocimiento de un tema que necesita ser explorado con mayor profundidad en el entorno nacional.

Tanto estudiantes como docentes se sienten motivados e interesados por incorporar más elementos de la investigación en su quehacer diario. Esto debes ser visto como una oportunidad, puesto al igual que en experiencias internacionales, se expresa la necesidad de incorporar la investigación y la ciencia de forma más contundente en la Terapia ocupacional. Se recomienda reforzar la formación de pregrado de Terapia ocupacional, vinculando los cursos de investigación con los intereses disciplinares y no sólo con el aprendizaje de metodologías de investigación de forma aislada (Benevides, Vause-Earland, \& Walsh, 2015). En ese sentido se hace interesante que los Terapeutas ocupacionales con conocimiento o interés en investigación científica puedan hacerse parte de los cursos de formación de pregrado vinculados a metodología e investigación, no sólo como sujetos aislados que realizan una recomendación temática específica.

A su vez también se ve como un área importante el capacitar y formar a los Terapeutas ya egresados en materias de investigación, puesto muchos podrían incorporar elementos de investigación en su práctica diaria, pudiendo evaluar sus intervenciones o generando nue- vos conocimientos que sean atingentes a la profesión desde la propia experiencia profesional.

\section{AgradeCimientos}

El equipo investigador le agradece a la Escuela de Terapia ocupacional de la Universidad Bernardo Óhiggins y a su directora Solange Balbontin Medel, por facilitar la ejecución de esta investigación (con materiales y espacio físico), y por su compromiso con el fortalecimiento de la investigación científica en Terapia ocupacional.

\section{REFERENCIAS BIBLIOGRÁFICAS}

American Occupational Therapy Association (2011). Occupational therapy research agenda. Am J Occup Ther, 65(6 Suppl.), S4-S7. http://dx.doi.org/10.5014/ajot.2011.65S4.

American Occupational Therapy Association. (2014). Research Agenda-Occupational therapy education research agenda. Am J Occup Ther, 68, S83-S86. http://dx.doi.org/10.5014/ajot.2014.685S06

Benevides, T.W., Vause-Earland, T., \& Walsh, R. (2015). Impact of a Curricular Change on Perceived Knowledge, Skills, and Use of Evidence in Occupational Therapy Practice: A Cohort Study. Am J Occup Ther, 69(2), 6912185010p1-9. doi: 10.5014/ajot.2015.018416.

Cohn, E.S., Coster, W.J., \& Kramer, J.M. (2014). Facilitated learning model to teach habits of evidence-based reasoning across an integrated master of science in occupational therapy curriculum. Am J Occup Ther, 68(2), S73-82. doi: 10.5014/ajot.2014.685S05.

du Toit, S.H., Wilkinson, AC., \& Adam, K. (2010). Role of research in occupational therapy clinical practice: applying action learning and action research in pursuit of evidence-based practice. Aust Occup Ther J, 57(5), 318-30. doi: 10.1111/j.1440-1630.2010.00851.x.

Fänge, A, \& Ivanoff, S.D. (2009). Integrating research into practice: a challenge for local authority occupational therapy. Scand J Occup Ther, 16(1), 40-8. doi: 10.1080/11038120802419357.

Gustafsson, L., Molineux, M., \& Bennett, S. (2014). Contemporary occupational therapy practice: The challenges of being evidence based and philosophically congruent. Aust Occup Ther J, 121-123. doi:10.1111/1440-1630.12110.

Karlsson, U., \& Törnquist, K. (2007). What do Swedish occupational therapists feel about research? A survey of perceptions, attitudes, intentions, and engagement. Scand J Occup Ther, 14(4), 221-9. doi: 10.1080/11038120601111049. 
Kiehofner, G. (2006). The Necessity of Research in a Profession. En G., Kielhofner, Research Occupational Therapy Practice: Method of Inquiry for Enhancing Practice (pp. 1-9). Philadelphia, EE.UU: F. A. Davis Company.

Lyons, C., Brown, T., Tseng, M.H., Casey, J., \& McDonald, R. (2011). Evidence-based practice and research utilisation: perceived research knowledge, attitudes, practices and barriers among Australian paediatric occupational therapists. Aust Occup Ther J, 58(3), 178-86. doi: 10.1111/j.1440-1630.2010.00900.x.

Thomas, A., Saroyan, A, \& Dauphinee, WD. (2011). Evidence-based practice: a review of theoretical assumptions and effectiveness of teaching and assessment interventions in health professions. Adv Health Sci Educ Theory Pract, 16(2), 253-76. doi: 10.1007/s10459010-9251-6.

Thomas, A., \& Law, M. (2013). Research utilization and evidence-based practice in occupational therapy: a scoping study. Am J Occup Ther, 67(4), e55-65. doi: 10.5014/ajot.2013.006395.

Thomas, A., \& Law, M.C. (2014). Evidence-based practice supports among Canadian occupational therapists. Can J Occup Ther, 81(2), 79-92.

Upton, D., Stephens, D., Williams, B., \& Scurlock-Evans, L. (2014). Occupational therapists' attitudes, knowledge, and implementation of evidence-based practice: a systematic review of published research. The British Journal of Occupational Therapy, 77(1), 24-38. doi:10.4276/030802214X13887685335544

Vogel, K. (2012). Librarians and occupational therapy faculty: A Collaboration for teaching evidence-based practice. Journal of Allied Health, 41(1), e15-20. 